\title{
Modular Estimation Strategy of Vehicle Dynamic Parameters for Motion Control Applications
}

\author{
Mustafa Rawash , Mohamed Abdelaziz ${ }^{1}$, Maged Ghoneima ${ }^{2}$ and Farid Tolbah $^{3}$ \\ ${ }^{1}$ Ain Shams University, Faculty of Engineering, Automotive Department, Cairo, Egypt \\ ${ }^{2}$ Ain Shams University, Faculty of Engineering, Mechatronics Department, Cairo, Egypt \\ ${ }^{3}$ Ain Shams University, Faculty of Engineering, Design and Production Department, Cairo, Egypt
}

\begin{abstract}
The presence of motion control or active safety systems in vehicles have become increasingly important for improving vehicle performance and handling and negotiating dangerous driving situations. The performance of such systems would be improved if combined with knowledge of vehicle dynamic parameters. Since some of these parameters are difficult to measure, due to technical or economic reasons, estimation of those parameters might be the only practical alternative. In this paper, an estimation strategy of important vehicle dynamic parameters, pertaining to motion control applications, is presented. The estimation strategy is of a modular structure such that each module is concerned with estimating a single vehicle parameter. Parameters estimated include: longitudinal, lateral, and vertical tire forces - longitudinal velocity - vehicle mass. The advantage of this strategy is its independence of tire parameters or wear, road surface condition, and vehicle mass variation. Also, because of its modular structure, each module could be later updated or exchanged for a more effective one. Results from simulations on a 14-DOF vehicle model are provided here to validate the strategy and show its robustness and accuracy.
\end{abstract}

\section{Introduction}

The presence of active safety systems, such as Antilock Braking, Traction Control and Stability Control systems, have become increasingly important. Such systems or motion control systems help maintain driver's control over the vehicle in dangerous driving situations, thereby reducing accident rates. Also such systems are capable of enhancing vehicle performance and handling.

The effectiveness of such control systems can be enhanced, if combined with knowledge of certain dynamic parameter. Some of these parameters are difficult to measure due to either technical or economic reasons, therefore, the only practical alternative is estimation of these parameters.

Typically, vertical tire forces are estimated using longitudinal and lateral load transfers, and static loads on each wheel [1-4]. In [5] Antonov et al. proposed an advanced method for calculating vertical tire forces based on modelled suspension system and using estimated forces acting on vehicle centre of gravity.

Longitudinal or lateral tire forces can be estimated based on analytical tire models, such as linear model [6], Dugoff model [7], or Magic Formula model [8]. However, such models rely on parameters that are specific to each tire and vary with tire aging or road surface condition. Alternatively, estimation of longitudinal tire forces based on modelling wheel rotational dynamics and vehicle longitudinal dynamics has been pursued in the literature $[1,9]$. In [1], Cho et al. defined a Lyapunov function based on wheel rotational dynamics, to estimate longitudinal tire force by satisfying the Lyapunov stability conditions. A random-walk model was also defined for combined (longitudinal and lateral) tire force estimation. In [10] and [11] a sliding mode observer is used.

Lateral tire forces are more difficult to estimate than longitudinal tire forces. A number of studies have tried to estimate the summation of lateral tire forces on front wheels and rear wheels using yaw-plane bicycle models $[1,2,12,13,14]$. The summation can be further distributed on left and right wheels using normal forces acting on each wheel.

Knowledge of vehicle mass is important to vehicle dynamic parameters estimation. Moreover, vehicle mass could change significantly with the number of passengers in it or the amount of luggage. In $[3,15]$, vehicle mass identification is made at rest using suspension deflection transducers, which are uncommon in vehicles without active or semi-active suspension. Many authors utilize the fact that vehicle mass directly affects the relationship between the net longitudinal force on the vehicle and its longitudinal acceleration to design vehicle mass estimators. In [16], Bae et al. proposed an averaging recursive least squares estimator that uses longitudinal force, acceleration, and GPS-based road grade measurements to determine vehicle mass and aerodynamic drag. In [17], Grieser proposed a similar averaging estimator in which aerodynamic drag is simulated online, rather than estimated, then subtracted 
from force measurements. In [18, 19], Vahidi et al. proposed a similar averaging estimator that does not require road grade measurements, rather estimates vehicle mass, drag, and road grade simultaneously. The system accommodates the time-varying nature of aerodynamic drag and road grade through multi-rate forgetting. Also in [20], Winstead and Kolmanovsky used an extended Kalman filter for estimating vehicle states including mass.

There exist several tire force estimation systems in the literature [1-5, 11-13, 21, 22]. However, most of them suffer from dependence on knowledge of vehicle mass $[1,4,5,10-13,21,22]$, dependence on suspension deflection transducers for estimating vehicle mass and body roll angle [2,3], or dependence on tire models which has the disadvantage mentioned earlier. Also most tire forces estimation systems found in the literature do not provide a unified structure for estimating tire forces in all directions. However, in [14], Rezaeian et al. presented a novel tire force estimation strategy. The strategy is of modular structure, where each module is concerned with estimating tire force in a specific direction, and each module could be further developed. Also the strategy has the advantage of independence of tire parameters or wear, as well as road surface condition and vehicle mass variation. Other parameters estimated in that strategy were vehicle mass and longitudinal velocity - body roll and pitch angles.

Motivated by the work in [14], this paper presents further enhancements to the aforementioned strategy as follows:

- Applying wheel slip ratio estimation for better evaluation of longitudinal velocity when wheels are slipping.

- Adopting a new vehicle mass estimation system; one that is more or equally effective, while requiring minimal instrumentation and computational cost.

For brevity of this paper, only developed modules will be discussed in details. For more information on the rest of the modules, please refer to [14]. The rest of this paper is structured as follows: Section-2 presents the outline of the modular estimation strategy with details on developed modules. Section-3 presents and discusses results of simulations carried to validate the proposed strategy. Finally, a conclusion is presented in Section-4.

\section{Proposed estimation strategy}

Figure 1 shows the outline of the proposed estimation strategy, and Table 1 lists all the symbols used in the figure (The subscript ij denotes each individual wheel). The modules of the estimation strategy are organized in a hierarchy, where each module relies on sensory signals and estimates from higher order modules.

Undeveloped modules are implemented as in [14] as follows:

- Each individual longitudinal tire force is estimated using an observer based on rotational wheel dynamics, and the force is estimated to satisfy a Lyapunov stability condition.
- Vertical tire forces on front and rear axles are first calculated using a 1-DOF pitch model and longitudinal and vertical acceleration measurements, then each front and rear axle forces are distributed between right and left tires using 1-DOF roll plane model and lateral and vertical acceleration measurements.

- Vertical tire forces estimation relies on estimates of body pitch and roll angles, which are estimated using observers similar to that initially proposed in [23] for body roll angle estimation, and later generalized in [14] for both body roll and pitch angles estimation. Body roll observer is based on 1-DOF roll plane model and relies on lateral acceleration roll rate measurements, while body pitch observer is based on 1-DOF pitch plane model and relies on longitudinal and pitch rate measurements.

- Lateral tire forces on front and rear axles are first estimated using an Unscented-Kalman-Filter as an observer for 3-DOF yaw plane bicycle model, where the forces are modelled as random walk states. Then each axle forces are distributed between right and left tires using vertical load distribution.

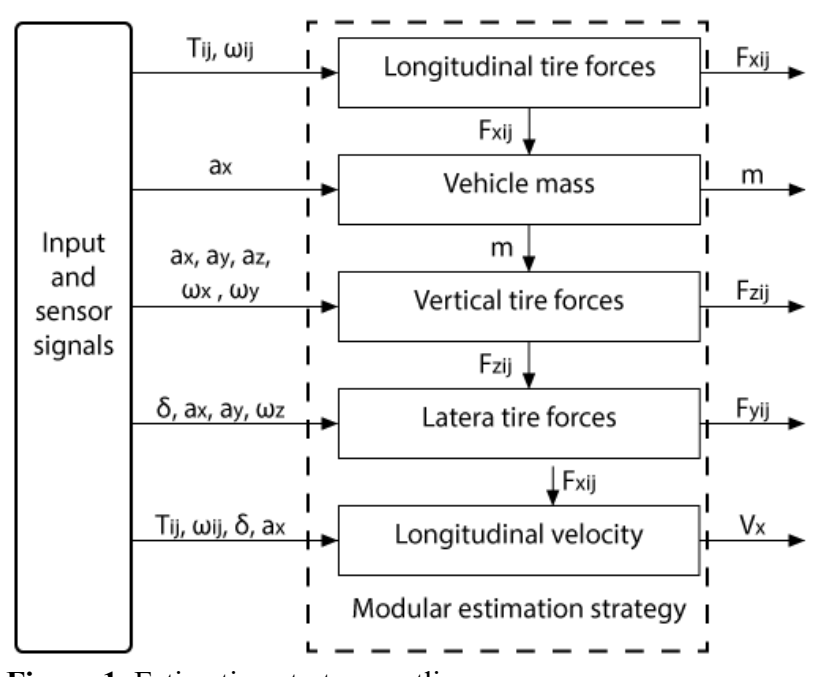

Figure 1. Estimation strategy outline.

Table 1. Definitions of symbols used in Figure 1.

\begin{tabular}{|c|c|}
\hline Symbol & Definition \\
\hline$T_{i j}$ & Wheel driving or braking torque \\
\hline$\omega_{i j}$ & Wheel angular velocity \\
\hline$\delta$ & Front steering angle \\
\hline$a_{x}$ & Measured longitudinal acceleration \\
\hline$a_{y}$ & Measured lateral acceleration \\
\hline$a_{z}$ & Measured vertical acceleration \\
\hline$\omega_{x}$ & Measured roll angular velocity \\
\hline$\omega_{y}$ & Measured pitch angular velocity \\
\hline$\omega_{z}$ & Measured yaw angular velocity \\
\hline$F_{x i j}$ & Estimated longitudinal tire forces \\
\hline$F_{y i j}$ & Estimated lateral tire forces \\
\hline$F_{z i j}$ & Estimated vertical tire forces \\
\hline$m$ & Estimated vehicle mass \\
\hline$V_{x}$ & Estimated longitudinal velocity \\
\hline
\end{tabular}




\subsection{Vehicle mass estimation}

In [24], Fathy et al. proposed a mass estimation system that relies on minimal instrumentation, as opposed to previous endeavours by authors where mass estimation relies on knowing vehicle aerodynamic characteristics or road grade angle [16,17]. Additionally, the proposed system is simpler than the ones presented by Vahidi et al. in $[18,19]$, Winstead and Kolmanovsky in [20]. Also in [24] the system was compared to those proposed by Bae et al. in [16] and Vahidi et al. in [18,19] to show faster and more accurate, reliable, and robust estimation.

The same system is applied here to estimate vehicle mass. It relies on the fact that inertial dynamics dominate the longitudinal behaviour of a vehicle at increasing frequencies. So when the vehicle motion is dominantly longitudinal the high frequency component of that motion obeys:

$$
m . \delta a_{x}=\delta F_{x}
$$

Where $m$ is the vehicle mass, $\delta a_{x}$ is the high frequency component of vehicle longitudinal acceleration and $\delta F_{x}$ is the high frequency component of the net longitudinal forces acting on the vehicle. Lead-lag filters are used to extract high frequency components of longitudinal acceleration measurement and total longitudinal tire forces estimate.

The first part of any vehicle journey usually includes enough longitudinal excitation, so mass estimation could be carried out only until change in estimated mass is less than a predefined value, as suggested in [14]. In [24], to insure that the vehicle motion is predominantly longitudinal, a supervisory algorithm was proposed, where mass estimation is only carried out when certain conditions are met.

\subsection{Longitudinal velocity estimation}

In [14] the linear velocity of each wheel centre is calculated from its angular velocity measurement, then transferred to vehicle centre of gravity using measured yaw rate. All 4 velocities are averaged to obtain an estimate of vehicle's longitudinal velocity. If the angular acceleration of a wheel is more than a predefined value, indicating that such wheel might be slipping, it is omitted from averaging.

Such technique of estimating longitudinal velocity doesn't provide accurate velocity estimate since angular acceleration is not a reliable indication of wheel slippage, each wheel is prone to a slight value of slippage which affects final velocity estimate, and slipping of all 4 wheels will result in the absence of velocity estimate.

In this paper, it is intended to combine such technique of longitudinal velocity estimation with wheel slip estimation to obtain a more accurate velocity estimate, where the linear velocity of each wheel centre shall be additionally calculated based on estimated slip ratio.

In [25] a system was proposed for estimating individual wheel slip ratio using Extended-Kalman-Filter (EKF) without detecting vehicle speed. The same technique shall be applied here but while using an Unscented-Kalman-Filter (UKF) instead.

The slip ratio can be defined as:

$$
\lambda=\frac{\omega \cdot r-v_{x}}{v_{x}}
$$

Where $\omega$ is the wheel angular velocity, $r$ is the wheel radius, assumed to be known, and $v_{x}$ is the wheel linear velocity at its center. From (2) the rate of change of slip ratio can be written as:

$$
\dot{\lambda}=\frac{\dot{\omega}}{\omega} \cdot(\lambda+1)-\frac{\dot{v}_{x}}{\omega \cdot r}(\lambda+1)^{2}=f(\lambda)
$$

Where $\dot{v}_{x}$ is the linear acceleration of the wheel centre, and is assumed in this paper to be equal to the measured longitudinal acceleration of the vehicle. However, a more accurate value may be obtained from longitudinal acceleration, lateral acceleration, and steering angle measurements.

The slip ratio shall be regarded as the state, and a discrete form of state update equation for the UKF can be derived using forward Euler discretization of (3) as follows:

$$
x_{k+1}=x_{k}+\Delta t . f\left(x_{k}\right)+w
$$

Where $x_{k+1}$ is the state at the current time step, $x_{k}$ is the state at the previous time step, $\Delta t$ is the sampling time, and $w$ is a process noise with zero mean and covariance $Q$.

The wheel rotational dynamics can be written as:

$$
T=F_{z} \cdot C(\lambda) \cdot r+I \cdot \dot{\omega}
$$

Where $T$ is the total driving or braking torque applied to the wheel, $F_{z}$ is the vertical tire force estimated, $C(\lambda)$ is the longitudinal friction coefficient as a function of slip ratio, and $I$ is the wheel moment of inertia.

In [25], $C(\lambda)$ was obtained from a Pacejka-Magic Formula tire model, which is against the strategy adopted in this paper because tire models rely on parameters that are specific to each tire and vary with tire aging or road surface condition. However, a modal representation of the coefficient of friction as a function of slip was applied, as shown in Figure 2, and later simulated on a wet road to verify its effectiveness.

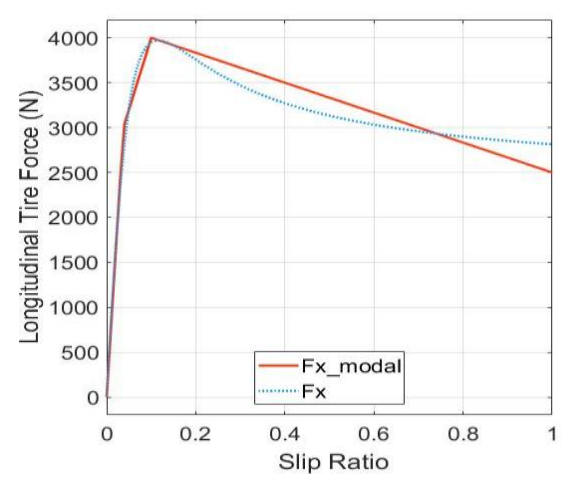

Figure 2. Modal representation of tire longitudinal force. 
The observation equation for the UKF is obtained from (5), and the total applied torque is regarded as the measurement.

$$
y_{k}=F_{z_{k}} \cdot C\left(x_{k}\right) \cdot r+I \cdot \dot{\omega}_{k}+v
$$

Where $v$ is a measurement noise with zero mean and covariance $R$. More information on UKF can be found in [26].

\section{Simulation results}

In [27], T. Shim and C. Ghike presented a 14-DOF vehicle model for vehicle dynamic simulations. The vehicle model was compared in 3 different simulated manoeuvres with 2 automotive industry standard Multibody simulation software; namely CarSim and ADAMS/Car, and has shown very close output.

The same 14-DOF model is adopted here for validating the presented estimation strategy. The vehicle model was equipped with Magic formula tire model found in [8] using TNO MF-Tire/MF-Swift 6.1 Tire Model Parameters, and implemented in MATLAB along with the estimation strategy. The vehicle model was simulated as a 4-wheel-drive in-wheel-motor electric vehicle, where each wheel is equipped with an electric motor capable of applying instant torque. The steering system was simulated as a simple front Ackerman steering system with steering ratio of $1 / 15$. For brevity of this paper more information on the vehicle or tire models and their parameters can be found in [27] and [8] respectively.

Sensory signals are needed for the estimation strategy, and include: longitudinal, lateral, and vertical accelerations (accelerometer), roll, pitch and yaw rate measurements (gyroscope), individual wheel rotational velocities, and front steering angle. All sensory signals were sampled from the vehicle model at a frequency of $100 \mathrm{~Hz}$, except for accelerations and body rotational velocities, which were additionally augmented with the effect of gravitational acceleration and sensor noise. The following equations describe the accelerometer and gyroscope sensor models.

$$
\begin{gathered}
\omega_{x_{m}}=\omega_{x}+\delta_{1} \\
\omega_{y_{m}}=\omega_{y}+\delta_{1} \\
\omega_{z_{m}}=\omega_{z}+\delta_{1} \\
a_{x_{m}}=\dot{u}+\omega_{y} \cdot w-\omega_{z} \cdot v-g \cdot \sin \theta+\delta_{2} \\
a_{y_{m}}=\dot{v}+\omega_{z} \cdot u-\omega_{x} \cdot w+g \cdot \cos \theta \cdot \sin \varphi+\delta_{2} \\
a_{z_{m}}=\dot{w}+\omega_{x} \cdot v-\omega_{y} \cdot u-g \cdot \cos \theta \cdot \cos \varphi+\delta_{2}
\end{gathered}
$$

Where $\omega_{x_{m}}, \omega_{y_{m}}$ and $\omega_{z_{m}}$ are the measured values of roll, pitch and yaw rates respectively with respect to body fixed frame, $\omega_{x}, \omega_{y}$ and $\omega_{z}$ are the actual roll, pitch and yaw rates respectively with respect to body fixed frame, $a_{x_{m}}, a_{y_{m}}$ and $a_{z_{m}}$ are measured longitudinal, lateral and vertical accelerations respectively with respect to body fixed frame, $u, v$ and $w$ are longitudinal, lateral and vertical velocities respectively with respect to body fixed frame, $g$ is the gravitational acceleration, $\theta$ and $\varphi$ are pitch and roll angles respectively of the body fixed frame with respect to world frame, and finally $\delta_{1}$ and $\delta_{2}$ are zero mean random noises with variances 0.01 and 0.0003475 respectively.

As in [14], 3 manoeuvres where simulated for validating the estimation strategy; slalom manoeuvre on a dry road, slalom manoeuvre on a wet road, and a DoubleLane-Change (DLC) manoeuvre on dry road. The coefficient of friction for a dry road is 1 , while for a wet road it's 0.6 , also an additional $200 \mathrm{Kg}$ were added to the actual vehicle mass in the slalom manoeuvre on wet road to evaluate the mass estimation module.

In all 3 manoeuvres, the vehicle is accelerated to $55 \mathrm{~km} / \mathrm{h}$ for the first 5 seconds, which is enough longitudinal excitation to obtain vehicle mass estimate. A switch was implemented to turn off mass estimation after the first 5 seconds have passed, and applying steering angles. Also in the last part of each manoeuvre the vehicle is decelerated.

The following subsections provide the simulation results of each manoeuvre, where the performance of each module is evaluated by calculating NormalizedRoot-Mean-Squared (NRMS) error, which is calculated according to the following equation:

$$
N R M S=\frac{\sqrt{\sum_{i=1}^{N}\left(A_{a c t}-A_{e s t}\right)^{2} / N}}{\max \left(\left|A_{a c t}\right|\right)}
$$

Where $A_{a c t}$ is the actual signal, $A_{\text {est }}$ is the estimated signal and $N$ is the number of collected samples. Also samples were collected at a frequency of $100 \mathrm{~Hz}$.

\subsection{Slalom manoeuvre on dry road}

Figure 3 shows the input torque, front steering angle, measured longitudinal acceleration, and measured lateral acceleration of the slalom manoeuvre on a dry road.

Figures 3-7 show the outputs of all estimation modules compared with their real values. Figure 7 shows that the mass estimate converges very closely to the real value, while the percentage error falls below $1 \%$ in less than 2.5 seconds. It is to be noted that an upper limit was imposed on mass estimate, and the saturation limit was $700 \mathrm{Kg}$ above original vehicle mass, and also in Figure 6 the estimated vertical tire forces are inaccurately estimated during the first 2.5 seconds, which is the time taken for the mass estimate to converge to an accurate value.

Also Figure 8 shows the longitudinal velocity estimate of the new proposed module to be more accurate, while the former estimation module deviates significantly during slippage. Table 2 lists the calculated NRMS of all estimates and the percentage error calculated for the mass estimate. 


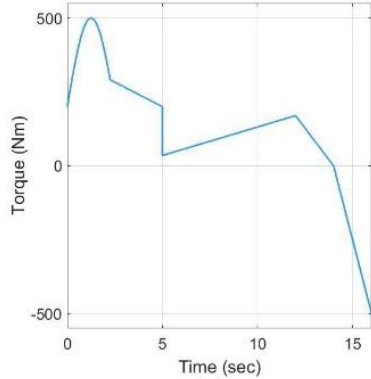

(a)

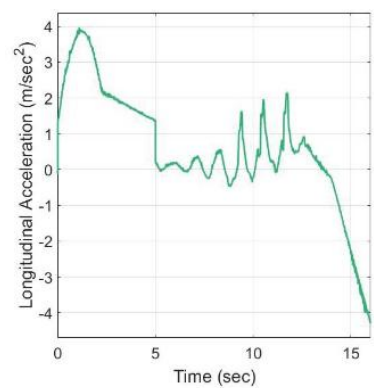

(c)

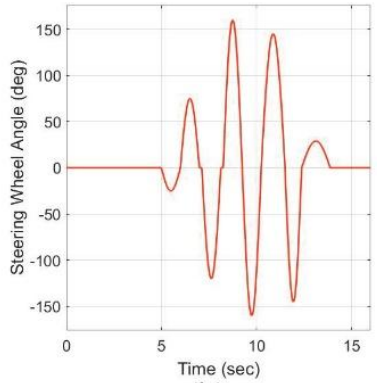

(b)

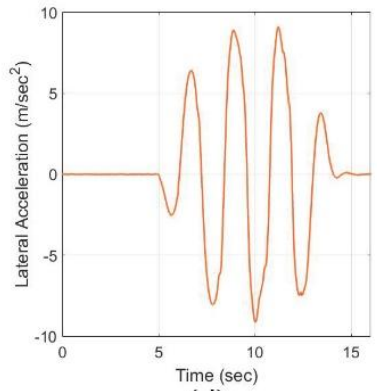

(d)
Figure 3. Slalom manoeuvre on dry road (a) input torque to each wheel (b) steering wheel angle (c) measured longitudinal acceleration (d) measured lateral acceleration.

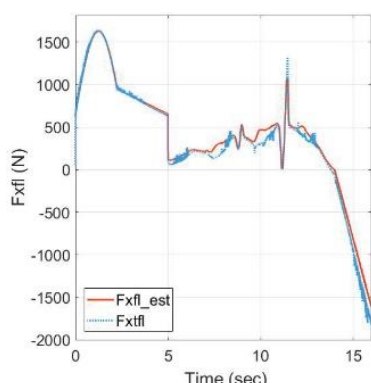

(a)

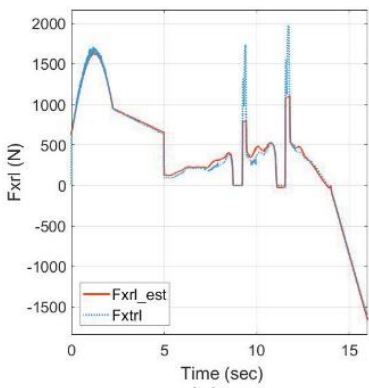

(c)

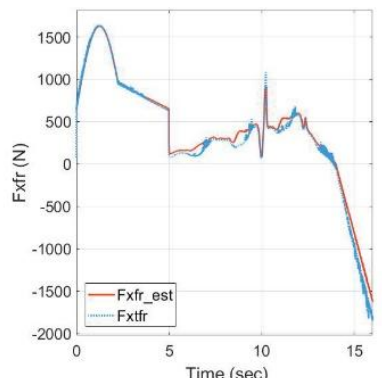

(b)

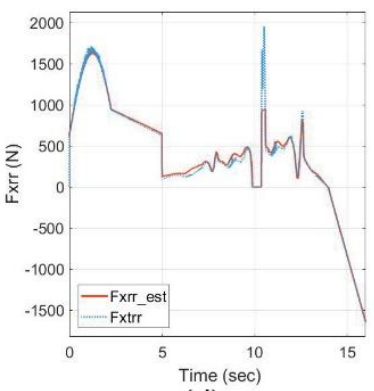

(d)
Figure 4. Estimated longitudinal forces in slalom manoeuvre on dry road (a) front-left (b) front-right (c) rear-left (d) rear-right. Fxtij denotes actual tire force, while Fxij_est denotes estimated tire force.

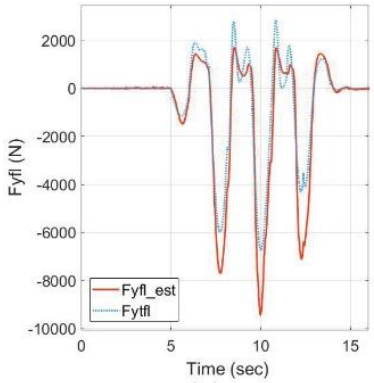

(a)

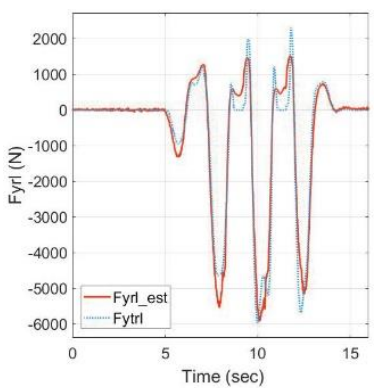

(c)

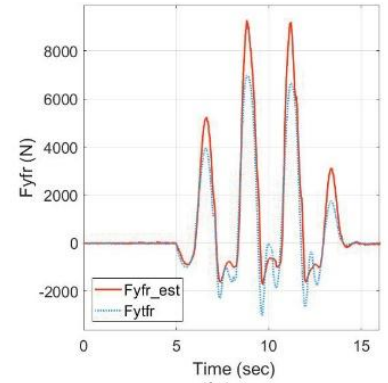

(b)

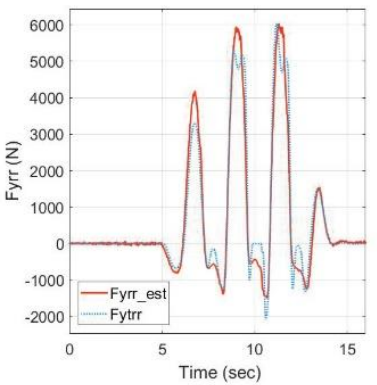

(d)
Figure 5. Estimated lateral forces in slalom manoeuvre on dry road (a) front-left (b) front-right (c) rear-left (d) rear-right. Fytij denotes actual tire force, while Fyij_est denotes estimated tire force.

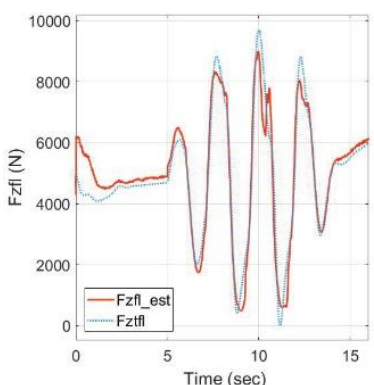

(a)

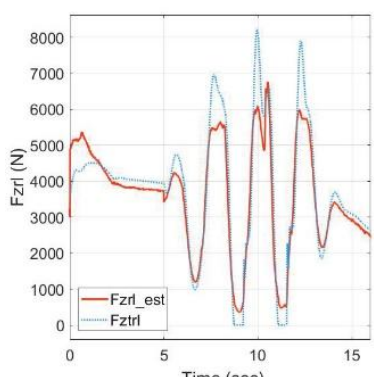

(c)

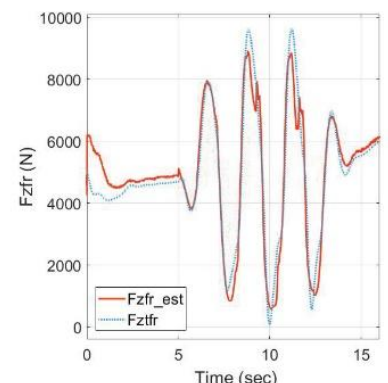

(b)

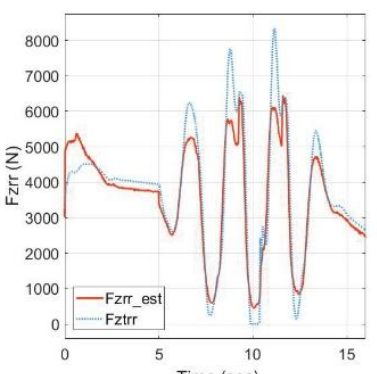

(d)
Figure 6. Estimated vertical forces in slalom manoeuvre on dry road (a) front-left (b) front-right (c) rear-left (d) rear-right. Fztij denotes actual tire force, while Fzij_est denotes estimated tire force. 


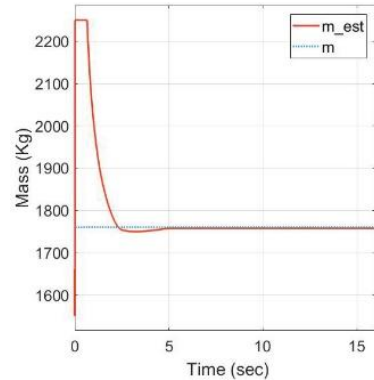

(a)

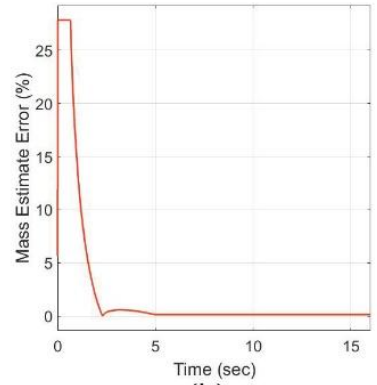

(b)
Figure 7. Mass estimation results in slalom manoeuvre on dry road. (a) comparison between actual mass $m$ and estimated mass m_est. (b) percentage error for mass estimation.

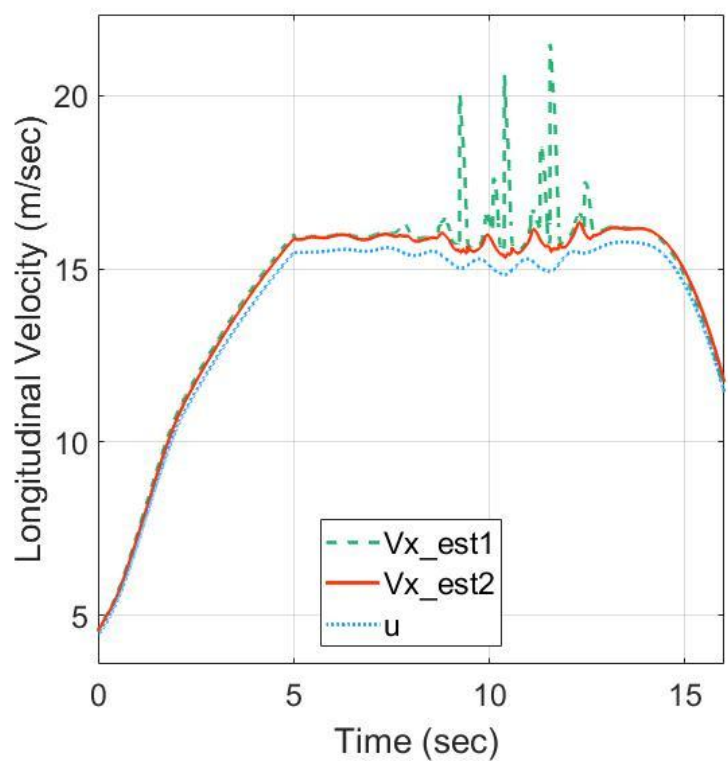

Figure 8. Longitudinal velocity estimation results in slalom manoeuvre on dry road. $u$ denotes actual vehicle velocity, Vx_est1 denotes velocity estimation without slip estimation, Vx_est2 denotes velocity estimation with slip estimation.

Table 2. NRMS and maximum values for all modules outputs in slalom manoeuvre on dry road.

\begin{tabular}{|c|l|l|l|l|l|l|}
\hline & & FL & FR & RL & RR & N/A \\
\hline \multirow{2}{*}{$F_{x}$} & NRMS & 4.29 & 4.17 & 5.2 & 4.19 & \\
\cline { 2 - 7 } & Max. & 1791 & 1794 & 1985 & 1953 & \\
\hline \multirow{2}{*}{$F_{y}$} & NRMS & 12.55 & 11.15 & 6.66 & 7.27 & \\
\cline { 2 - 7 } & Max. & 6727 & 6993 & 5979 & 6018 & \\
\hline \multirow{2}{*}{$F_{z}$} & NRMS & 5.9 & 6.22 & 7.45 & 7.33 & \\
\cline { 2 - 7 } & Max. & 9693 & 9621 & 8218 & 8336 & \\
\hline \multirow{2}{*}{$V_{x}$} & NRMS & & & & & 2.69 \\
\cline { 2 - 7 } & Max. & & & & & 15.77 \\
\hline \multirow{2}{*}{$\mathrm{m}$} & PE & & & & & 0.13 \\
\hline
\end{tabular}

\subsection{Slalom manoeuvre on wet road}

Figure 9 shows the input torque, front steering angle, measured longitudinal acceleration, and measured lateral acceleration of the slalom manoeuvre on a wet road.

Figures 10-14 show the outputs of all estimation modules compared with their real values. Figure 13 shows that the mass estimate converges very closely to the real value which is $200 \mathrm{Kg}$ more than in manoeuvres on dry road, while the percentage error falls to about $2 \%$ in less than 2.5 seconds and eventually remains below $2 \%$. Also Fig. 14 shows the new longitudinal velocity module still maintaining a more accurate output than the former estimation module. Table 3 lists all calculated NRMS values and percentage error for mass estimate.

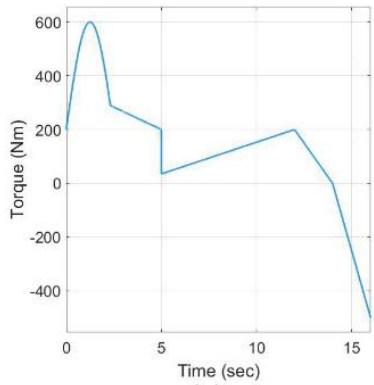

(a)

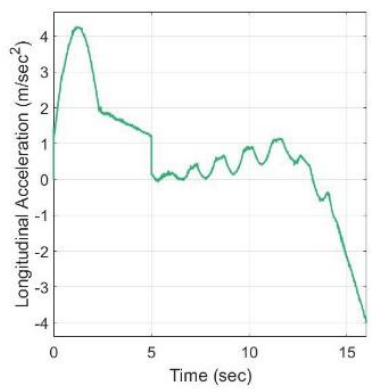

(c)

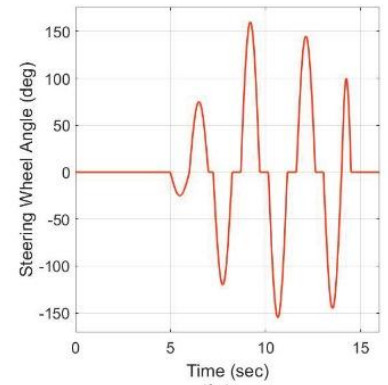

(b)

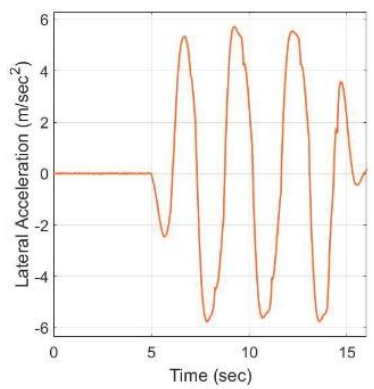

(d)
Figure 9. Slalom manoeuvre on wet road (a) input torque to each wheel (b) steering wheel angle (c) measured longitudinal acceleration (d) measured lateral acceleration.

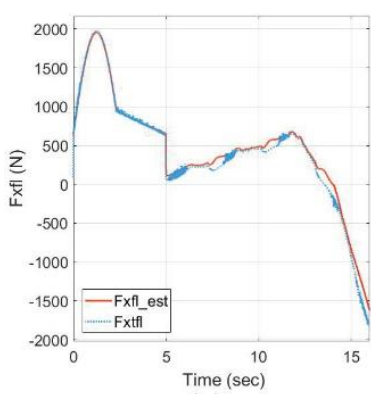

(a)

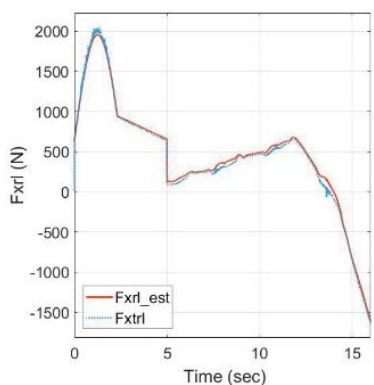

(c)

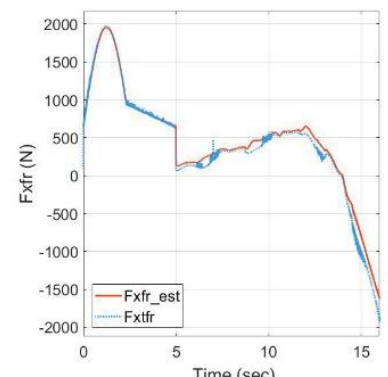

(b)

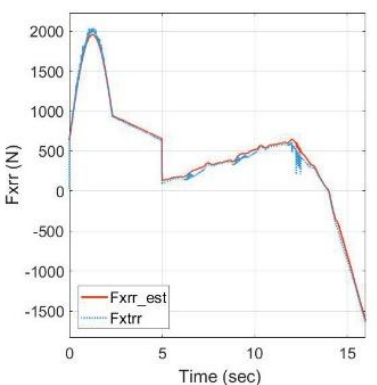

(d)
Figure 10. Estimated longitudinal forces in slalom manoeuvre on wet road (a) front-left (b) front-right (c) rear-left (d) rearright. Fxtij denotes actual tire force, while Fxij_est denotes estimated. 


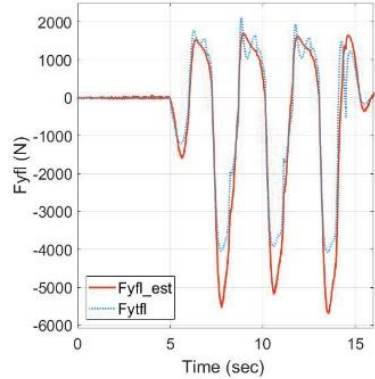

(a)

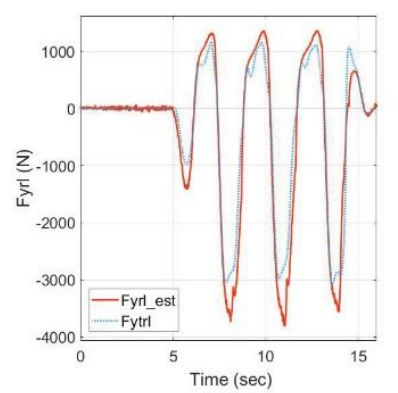

(c)

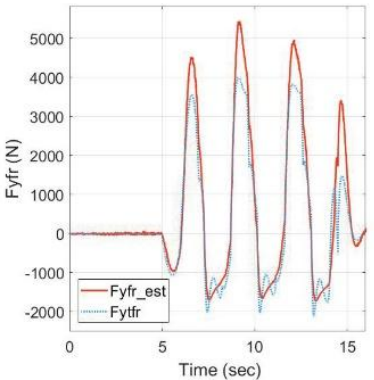

(b)

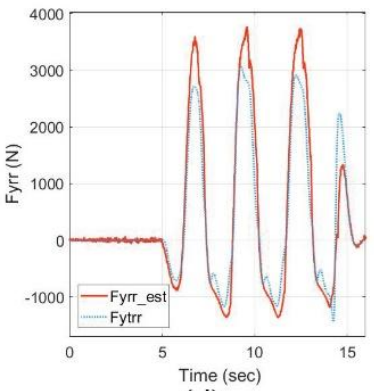

(d)
Figure 11. Estimated lateral forces in slalom manoeuvre on wet road (a) front-left (b) front-right (c) rear-left (d) rear-right. Fytij denotes actual tire force, while Fyij_est denotes estimated tire force.

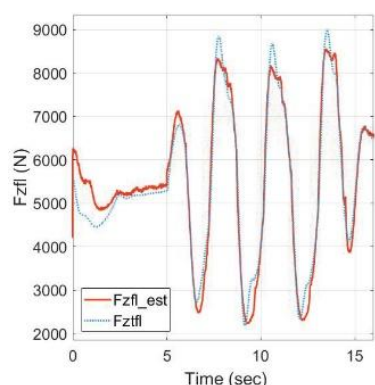

(a)

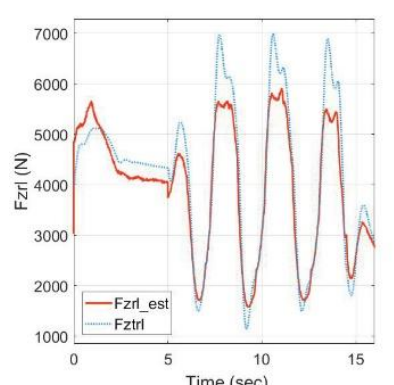

(c)

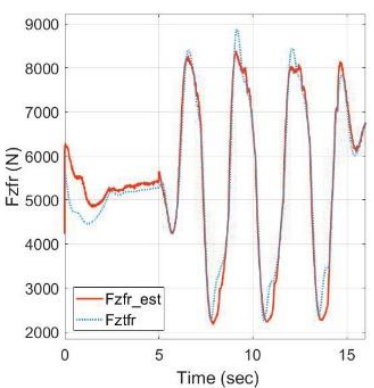

(b)

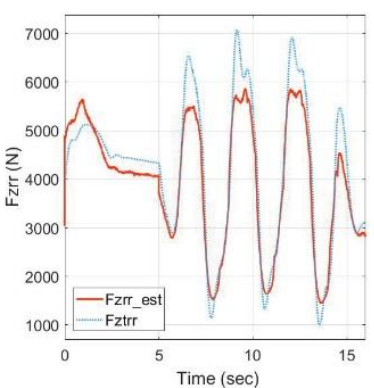

(d)
Figure 12. Estimated vertical forces in slalom manoeuvre on wet road (a) front-left (b) front-right (c) rear-left (d) rear-right. Fztij denotes actual tire force, while Fzij_est denotes estimated tire force.

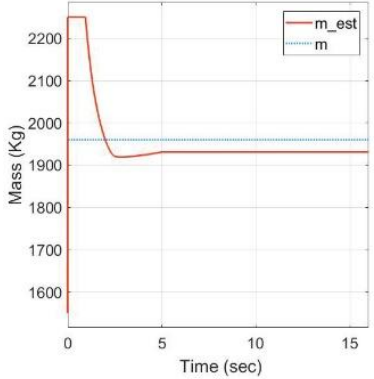

(a)

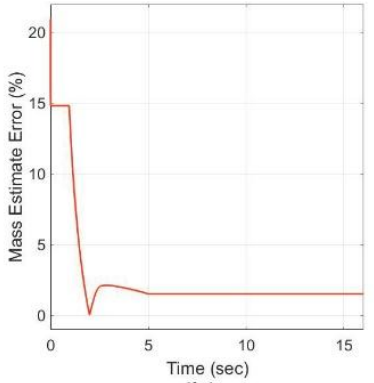

(b)
Figure 13. Mass estimation results for slalom manoeuvre on wet road. (a) comparison between actual mass $\mathrm{m}$ and estimated mass m_est. (b) percentage error for mass estimation.

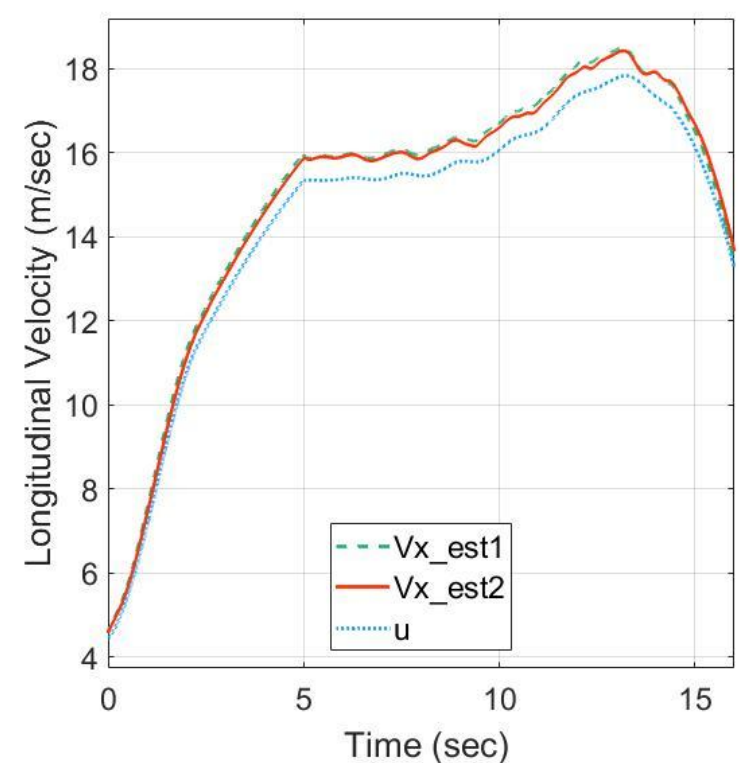

Figure 14. Longitudinal velocity estimation results in slalom manoeuvre on wet road. u denotes actual vehicle velocity, Vx_est1 denotes velocity estimation without slip estimation, $\mathrm{Vx}$ est2 denotes velocity estimation with slip estimation.

Table 3. NRMS and maximum values for all modules outputs in slalom manoeuvre on wet road.

\begin{tabular}{|l|l|l|l|l|l|l|}
\hline & & FL & FR & RL & RR & N/A \\
\hline \multirow{2}{*}{$F_{x}$} & NRMS & 4.07 & 4.24 & 2.09 & 2.15 & \\
\cline { 2 - 7 } & Max. & 1973 & 1973 & 2034 & 2034 & \\
\hline \multirow{2}{*}{$F_{y}$} & NRMS & 13.08 & 13.43 & 12.13 & 13.63 & \\
\cline { 2 - 7 } & Max. & 4079 & 4011 & 3083 & 3065 & \\
\hline \multirow{2}{*}{$F_{z}$} & NRMS & 4.01 & 4.16 & 7.02 & 6.7 & \\
\cline { 2 - 7 } & Max. & 8989 & 8883 & 6999 & 7080 & \\
\hline \multirow{2}{*}{$V_{x}$} & NRMS & & & & & 2.73 \\
\cline { 2 - 7 } & Max. & & & & & 17.83 \\
\hline \multirow{2}{*}{$\mathrm{m}$} & PE & & & & & 1.49 \\
\hline
\end{tabular}

\subsection{DLC manoeuvre on dry road}

Figure 15 shows the input torque, front steering angle, measured longitudinal acceleration, and measured lateral acceleration of the DLC manoeuvre on a dry road. 


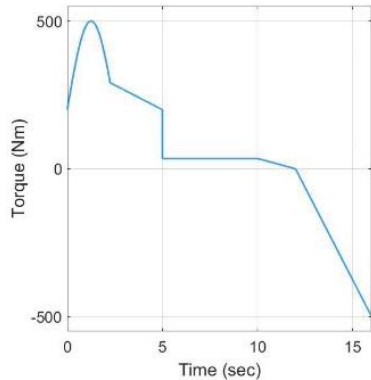

(a)

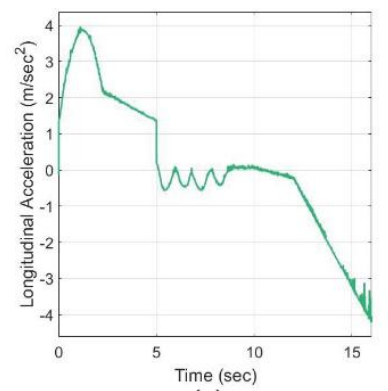

(c)

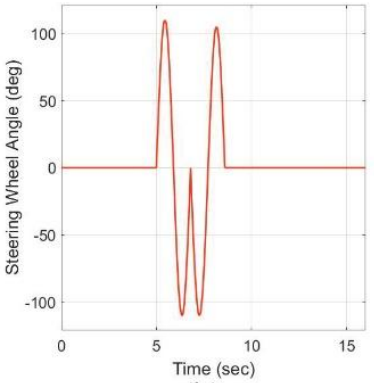

(b)

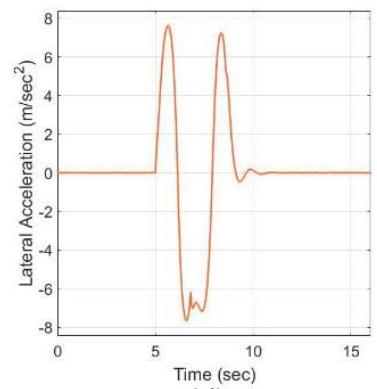

(d)
Figure 15. DLC manoeuvre (a) input torque to each wheel (b) steering wheel angle (c) measured longitudinal acceleration (d) measured lateral acceleration.

Figures 16-20 show the outputs of all estimation modules compared with their real values. Figure 19 shows the mass estimation module output to be exactly the same as in the slalom manoeuvre on dry road, while Figure 20 shows the new longitudinal velocity module to be still performing well. Table 4 lists all calculated NRMS values and percentage error for mass estimate.

The accuracy and performance of all undeveloped modules in all 3 manoeuvres is comparable to that found in [14].

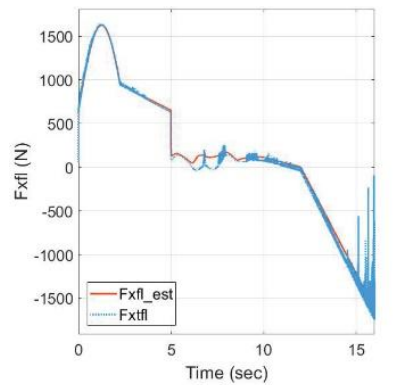

(a)

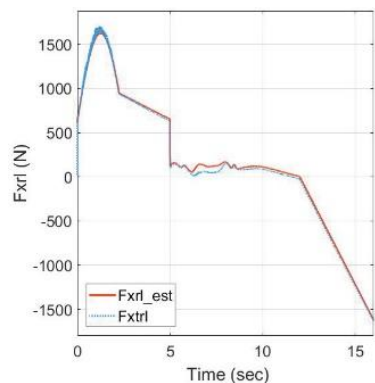

(c)

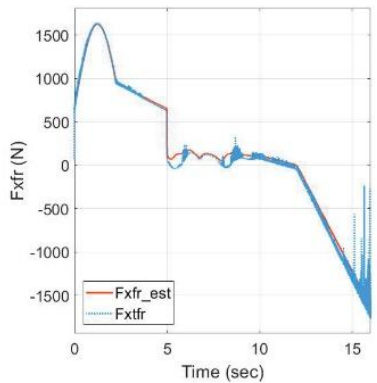

(b)

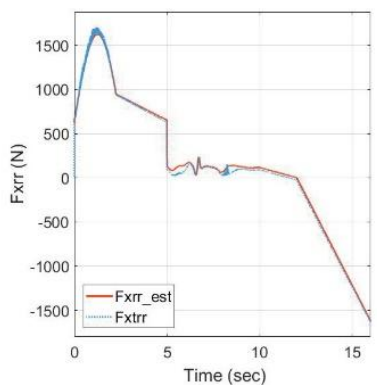

(d)
Figure 16. Estimated longitudinal forces in DLC manoeuvre (a) front-left (b) front-right (c) rear-left (d) rear-right. Fxtij denotes actual tire force, while Fxij_est denotes estimated.

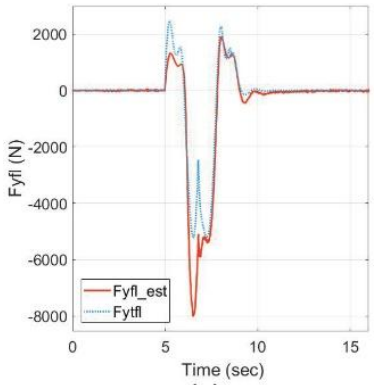

(a)

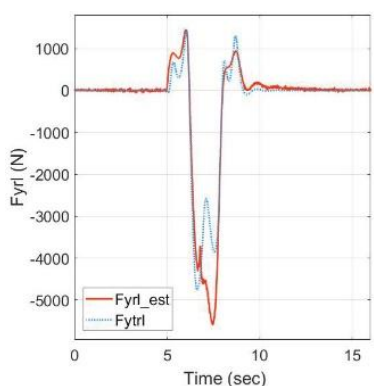

(c)

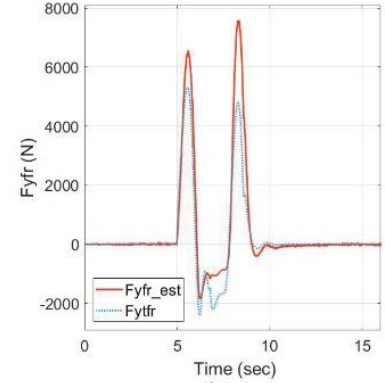

(b)

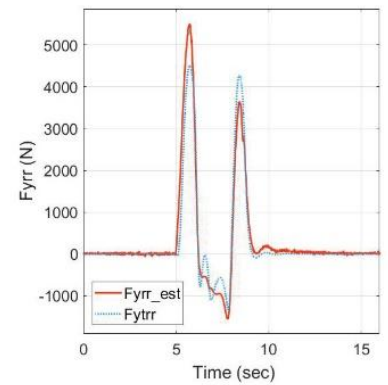

(d)
Figure 17. Estimated lateral forces in DLC manoeuvre (a) front-left (b) front-right (c) rear-left (d) rear-right. Fytij denotes actual tire force, while Fyij_est denotes estimated tire force.

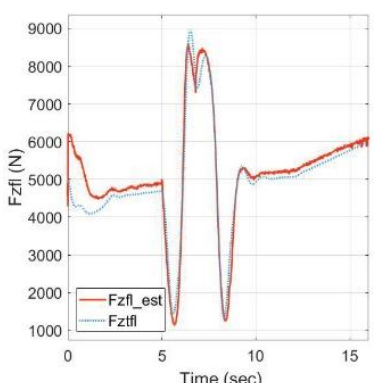

(a)

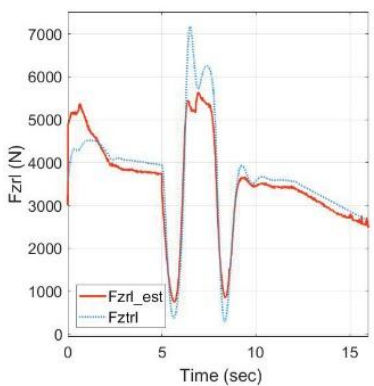

(c)

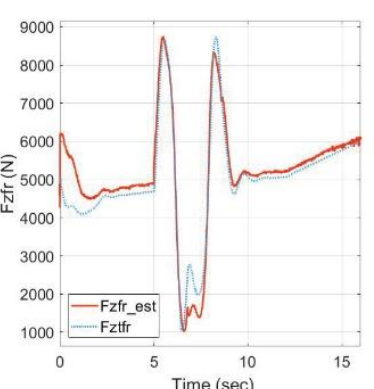

(b)

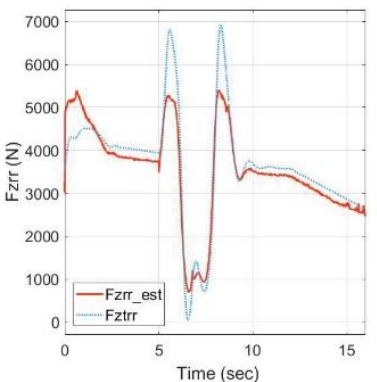

(d)
Figure 18. Estimated vertical forces in DLC manoeuvre (a) front-left (b) front-right (c) rear-left (d) rear-right. Fztij denotes actual tire force, while Fzij_est denotes estimated tire force. 


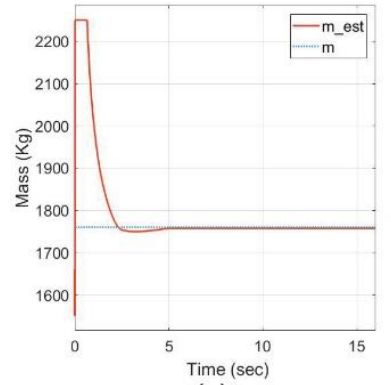

(a)

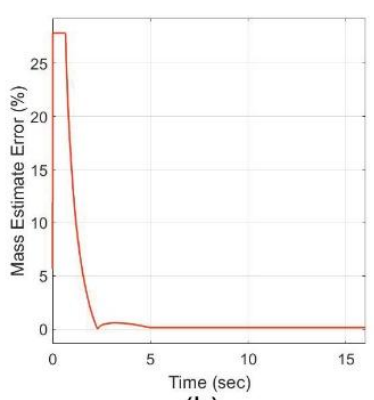

(b)
Figure 19. Mass estimation results for DLC manoeuvre. (a) comparison between actual mass $\mathrm{m}$ and estimated mass m_est. (b) percentage error for mass estimation.

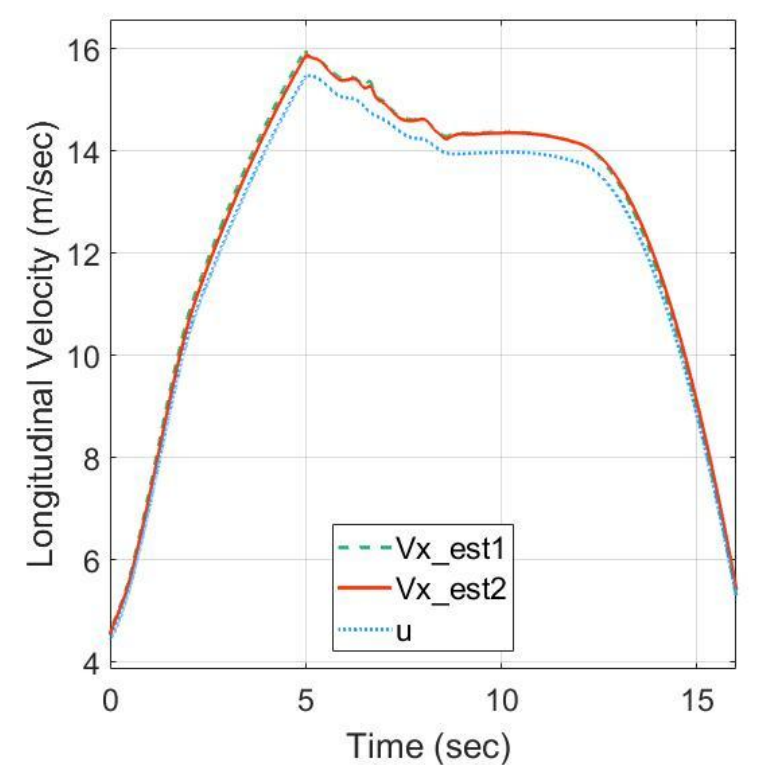

Figure 20. Longitudinal velocity estimation results in DLC manoeuvre. u denotes actual vehicle velocity, Vx_est1 denotes velocity estimation without slip estimation, Vx_est 2 denotes velocity estimation with slip estimation.

Table 2. NRMS and maximum values for all modules outputs in DLC manoeuvre.

\begin{tabular}{|l|l|l|l|l|l|l|}
\hline & & FL & FR & RL & RR & N/A \\
\hline \multirow{2}{*}{$F_{x}$} & NRMS & 3.87 & 3.81 & 2.14 & 2.09 & \\
\cline { 2 - 7 } & Max. & 1728 & 1738 & 1683 & 1683 & \\
\hline \multirow{2}{*}{$F_{y}$} & NRMS & 10.83 & 11.96 & 9.11 & 6.91 & \\
\cline { 2 - 7 } & Max. & 5229 & 5331 & 4756 & 4506 & \\
\hline \multirow{2}{*}{$F_{z}$} & NRMS & 4.72 & 5.1 & 6.22 & 6.76 & \\
\cline { 2 - 7 } & Max. & 8955 & 8740 & 7167 & 6926 & \\
\hline \multirow{2}{*}{$V_{x}$} & NRMS & & & & & 2.12 \\
\cline { 2 - 7 } & Max. & & & & & 15.47 \\
\hline $\mathrm{m}$ & PE & & & & & 0.1335 \\
\hline
\end{tabular}

\section{Conclusion}

The presence of active safety systems, such as Antilock Braking, Traction Control and Stability Control systems, have become increasingly important. The performance of such control systems depends on knowledge of vehicle dynamic parameters which are difficult to measure directly due to economic or technical reasons.
In [14] a novel unified estimation strategy was proposed for estimating tire forces in all 3 directions. The estimation strategy was of modular structure and has the advantage of being robust to vehicle mass change, since it includes mass estimation, and relies on no tire model, as tire models are prone to uncertainty due to tire aging or road surface condition.

Motivated by the work in [14] a modular estimation strategy for dynamic parameters pertaining to motion control applications is developed and presented, based on previous modules from [14] and 2 developed modules for vehicle mass estimation and longitudinal velocity estimation.

A 14-DOF vehicle model was simulated on Matlab in 3 manoeuvres, namely, Slalom manoeuvre on dry road, Slalom manoeuvre on wet road with additional $200 \mathrm{Kg}$ of mass, and Double-Lane-Change manoeuvre on a dry road. Simulation results shows good performance for the newly developed modules.

The new developed mass estimation module shows good performance while being simpler, relying only on minimal instrumentation.

Even though the new longitudinal velocity estimation relied on a modal tire model, it maintained an accurate output on varying road condition. The velocity estimate could be useful for motion control applications such as Active Cruise Control.

\section{References}

1. W. Cho, J. Yoon, S. Yim, B. Koo, K. Yi, "Estimation of Tire Forces for Application to Vehicle Stability Control", IEEE Trans. Veh. Technol., 59, 2, (2010).

2. M. Doumiati, A. Victorino, D. Lechner, G. Baffet, A. Charara, "Observers for vehicle tyre/road forces estimation: experimental validation", Veh. Syst. Dyn., 48, 11, (2010).

3. M. Doumiati, A. Victorino, A. Charara, D. Lechner, "Lateral load transfer and normal forces estimation for vehicle safety: experimental test", Veh. Syst. Dyn., 47, 12, (2009).

4. A. Rabhi, N.K. M'Sirdi, A. Elhajjaji, "Estimation of Contact Forces and Tire Road Friction", Proc. Mediterranean Conf. Control Autom., 1-6, (2007).

5. S. Antonov, A. Fehn, A. Kugi, "Unscented Kalman filter for vehicle state estimation", Veh. Syst. Dyn., 49, 9, (2011).

6. J. Kim, H. Lee, S. Choi, “A robust road bank angle estimation based on a proportional-integral $\mathrm{H} \infty$ filter", Proc. Inst. Mech. Eng. D. J. Autom. Eng., 226, 6, (2012).

7. R. Ghandour, A. Victorino, M. Doumiati, A. Charara, "Tire/road friction coefficient estimation applied to road safety", Proc. 18th Mediterranean Conf. Control Autom., 1485-1490, (2010).

8. H.B. Pacejka, I. Besselink, Tire and Vehicle Dynamics, (Elsevier, Butterworth-Heineman 2012).

9. R. Rajamani, D. Piyabongkarn, J.Y. Lew, J.A. Grogg, "Algorithms for Real-Time Estimation of Individual 
Wheel Tire-Road Friction Coefficients", Proc. Amer. Control Conf., 4682-4687, (2006).

10. B. Jaballah, N.K. M'Sirdink, A. Naamane, H. Messaoud, "Estimation of vehicle longitudinal tire force with FOSMO \& SOSMO", IJ-STA, 5, 1, (2011).

11. H. Imine, N.K. M'Sirdi, Y. Delanne, "Adaptive Observers and Estimation of the Road Profile", Soc. Automotive Eng. World Cong., (2003).

12. L.R. Ray, "Nonlinear State and Tire Force Estimation for Advanced Vehicle Control", IEEE Trans. Control Syst. Technol., 3, 1, (1995).

13. K. Huh, C. Seo, J. Kim, D. Hong, "Active Steering Control Based on The Estimated Tire Forces", Proc. Amer. Control Conf., 729-733, (1999).

14. A. Rezaeian, R. Zarringhalam, S. Fallah, W. Melek, A. Khajepour, S.K. Chen, N. Moshchuck, B. Litkouhi, "Novel Tire Force Estimation Strategy for Real-Time Implementation on Vehicle Applications", IEEE Trans. Veh. Technol., 64, 6, (2015).

15. M. Doumiati, A. Victorino, A. Charara, D. Lechner, "Virtual sensors, application to vehicle tire-road normal forces for road safety", Proc. Amer. Control Conf., 3337-3343, (2009).

16. H.S. Bae, J. Ryu, J.C. Gerdes, "Road Grade and Vehicle Parameter Estimation for Longitudinal Control Using GPS", IEEE Proc. Intel. Transp. Syst. Conf., (2001).

17. J. Grieser, "Method for Determining an Estimate of the Mass of a Motor Vehicle", U.S. Patent, $6,980,900,(2005)$.

18. A. Vahidi, A. Stefanopoulou, H. Peng, "Experiments for Online Estimation of Heavy Vehicle's Mass and Time-Varying Road Grade", Proc. ASME IMECE, 451-458, (2003).

19. A. Vahidi, M. Druzhinina, A. Stefanopoulou, H. Peng, "Simultaneous Mass and Time-Varying Grade Estimation for Heavy-Duty Vehicles", Proc. Amer. Control Conf., 4951-4956, (2003).

20. V. Winstead, I.V. Kolmanovsky, "Estimation of Road Grade and Vehicle Mass via Model Predictive Control", Proc. IEEE Conf. Control App., 1588-1593, (2005).

21. M.A. Wilkin, W.J. Manning, D.A. Crolla, M.C. Levesley, "Use of an extended Kalman filter as a robust tyre force estimator", Veh. Syst. Dyn., 44, 5059, (2006).

22. J. Stephant, A. Charara, D. Meizel, "Virtual Sensor: Application to Vehicle Sideslip Angle and Transversal Forces", IEEE Trans. Ind. Electron., 51, 2, (2004).

23. A. Rehm, "Estimation of Vehicle Roll Angle", Proc. $4^{\text {th }}$ ISCCSP, 1-5, (2010).

24. H.K. Fathy, D. Kang, J.L. Stein, "Online Vehicle Mass Estimation Using Recursive Least Squares and Supervisory Data Extraction", Proc. Amer. Control Conf., 1842-1848, (2008).

25. Y. Zhang, H. Zhao, L. Yuan, H. Chen, "Slip Ratio Estimation for Electric Vehicle with In-wheel Motors based on EKF without Detection of Vehicle Velocity", Proc. 28th CCDC, 4427-4432, (2016).

26. S. Julier, J. Uhlmann, H.F. Durrant-Whyte, "A New Method for the Nonlinear Transformation of Means and Covariances in Filters and Estimators", IEEE Trans. Autom. Control, 45, 3, (2000).

27. T. Shim, C. Ghike, "Understanding the limitations of different vehicle models for roll dynamics studies", Veh. Syst. Dyn., 45, 3, (2007). 